Corporación Universitaria del Caribe - CECAR ISSN: 2422 - 085X

Enero - Diciembre 2015

Sincelejo, Colombia
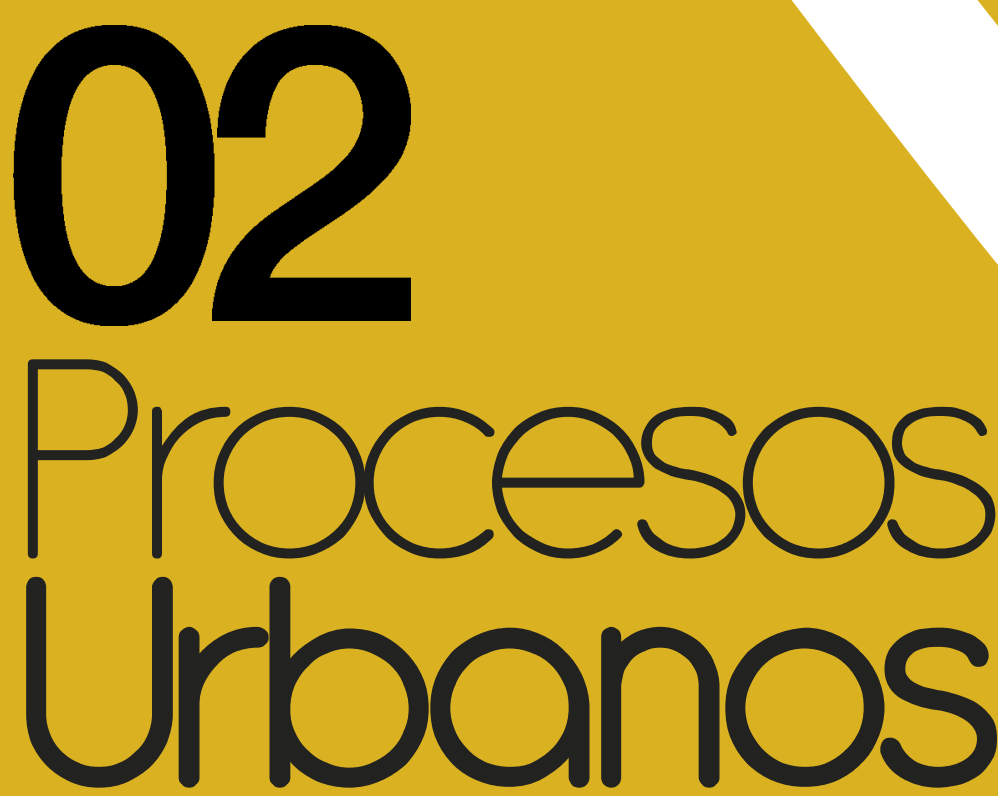

Revista de divulgación científica

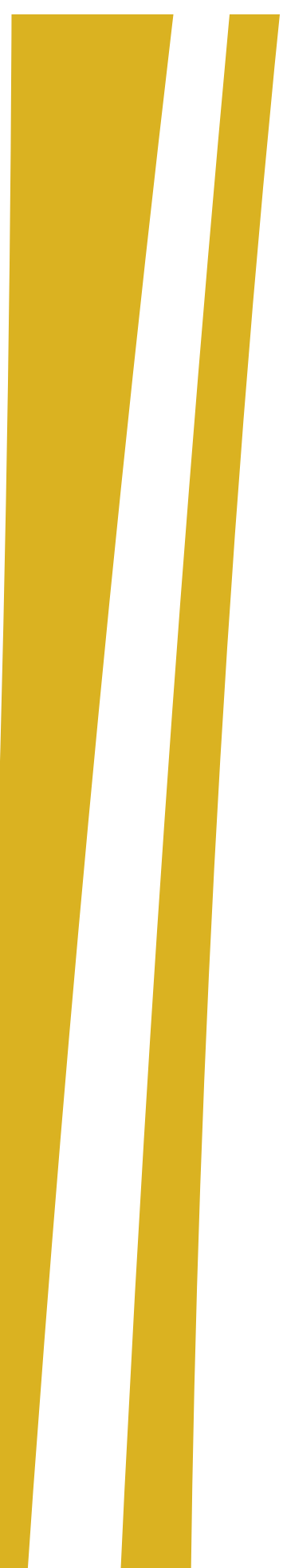

Facultad de Ciencias Básicas, Ingeniería y Arquitectura

Grupo de investigación: Teoría e historia de la arquitectua y la ciudad. 

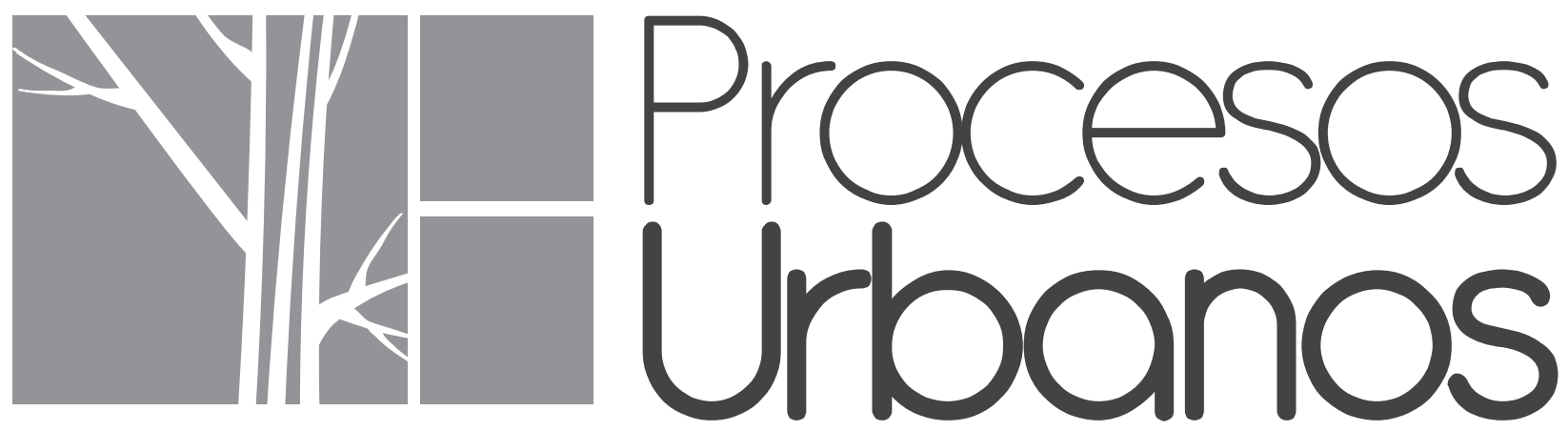

Revista de divulgación científica

Facultad de Ciencias Básicas, Ingeniería y Arquitectura. Grupo de investigación: Teoría e historia de la arquitectura y la ciudad 
Revista Procesos Urbanos

Facultad de Ciencias Básicas, Ingeniería y Arquitectura.

ISSN: 2422-085X (papel)

ISSN: $2500-5200$ (on line)

Sincelejo, enero - diciembre de 2015

\section{Piedad Martínez Carazo}

Rectora

\section{Lidia Flórez de Albis}

Vicerrectora académica

Jhon Víctor Vidal

Vicerrector de Ciencia, Tecnología e Innovación

\section{Pedro Arturo Martínez Osorio}

Editor

\section{Gilberto Martínez Osorio \\ Francisco Martínez Ochoa \\ Coeditores}

\section{Comité Editorial:}

PhD. Alexander Niño Soto. Universidad del Norte

PhD. Jorge Ramírez Nieto. Universidad Nacional de Colombia

Msc. Juan Carlos Pergolis. Universidad Católica de Colombia

PhD. Piedad Martínez Carazo. Corporación Universitaria del Caribe, CECAR

PhD. Emiro F. Martínez. Universidad de York, Canadá

\section{Comité Científico:}

Msc. Gloria Aponte García. UPB, Medellín

PhD. Jorge Gómez Ricardo, Universidad del Magdalena

Msc. Gilberto Martínez Osorio, Corporación Universitaria del Caribe, CECAR

PhD. Alfredo Otero Ortega, Corporación Universitaria del Caribe, CECAR

\section{Editorial CECAR}

Libia Narváez Barbosa

Coordinadora editorial

\section{Eduardo Támara Galván \\ Corrector de estilo}

\section{GRÁFICAS DEL CARIBE S.A.S.}

Diseño gráfico

Cra. 1B No. 40-42 Montería Tel. (57) (4) 7826622 Telefax (57) (4) 7817112

Email: diseno@graficaribe.co

Dirección:

Carretera Troncal de occidente

Kilómetro 1 vía a Corozal

Tel: 280402928040172804018 Ext 1115

www.cecar.edu.co

http://revistas.cecar.edu.co/procesos-urbanos

http://ojs.cecar.edu.co/procesos-urbanos

PROCESOS URBANOS hace parte de:

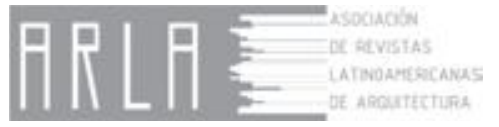




\title{
EL PAPEL DE LA FAMILIA Y LA CULTURA EN LA TRANSFORMACIÓN DEL HABITAT: HABITAT Y FAMILIAS EN LA CIUDAD DE SINCELEJO: 1950-2000
}

The role of family and culture in the transformation of habitat: habitat and families in the city of sincelejo: $1950-2000$

\author{
Angélica María Sierra Franco ${ }^{1}$
}

Enviado 05 de junio de 2015. Aceptado: 16 de septiembre de 2015

\section{RESUMEN}

La familia constituye una suerte de sistema operativo (reproductivo, laboral, y cultural) de la sociedad. En virtud de esta condición, los modos de comportamiento de sus miembros articulan la familia al territorio, incidiendo en la formación, desarrollo o desintegración de los lugares y figuras espaciales del hábitat, especialmente el hábitat residencial: la casa. Esas figuras están íntimamente ligadas y condicionadas por la evolución, las crisis y las tendencias de la sociedad, de manera que constituyen así la red social primaria para el ser humano en cualquier etapa de la vida. La importancia de este estudio radica en que la composición, organización y movimientos del núcleo familiar, ofrecen una gama muy diversa de variables de análisis e interpretaciones, para comprender su incidencia en la formación del hábitat, donde las agrupaciones familiares se asocian a otras modalidades de agrupación: vecinal, comunitaria, territorial, económica, social e institucional, y ya sea que se mantengan estables o que sufran cambios profundos, permanecen a lo largo del tiempo como factor que configura sus distintos modos de habitar el territorio.

Palabras clave: Comunidad familiar, hábitat residencial, vivienda, cultura, costa Caribe colombiana.

\begin{abstract}
The family is a kind of (reproductive, labor and cultural) operating system of society. Under this condition, the modes of behavior of family members articulate the territory, focusing on the formation, development or disintegration of places and spatial figures habitat, especially the residential habitat: the house. These figures are closely linked and conditioned by evolution, crisis and trends in society, so that social network are so primary to humans at any stage of life. The importance of this study is that the composition, organization and movements of the family, offer a diverse range of variables for the analysis and interpretation to understand its impact on habitat formation, where family groups are associated with other forms of grouping: neighborhood, community, territorial, economic, social and institutional, and either remain stable or undergo profound changes, remain throughout time as a factor shaping their different ways of inhabiting the territory.
\end{abstract}

Keywords: family community, residential housing, housing, culture, Caribbean coast of Colombia.

${ }^{1}$ Arquitecta, especialista en gerencia ambiental, candidata a magíster en habitat. Docente tiempo completo programa de arquitectura de Cecar. Email : angelica.sierraf@cecar.edu.co 
PROCESOS URBANOS - Revista de divulgación científica vol. 2 enero - diciembre 2015 (107 - 116)

\section{INTRODUCCIÓN}

Úrsula se dio cuenta de pronto que la casa se había llenado de gente, que sus hijos estaban a punto de casarse y tener hijos, y que se verían obligados a dispersarse por falta de espacio. Entonces sacó el dinero acumulado en largos años de dura labor, adquirió compromisos con sus clientes y emprendió la ampliación de la casa.

Dispuso que se construyera una sala formal para las visitas, otra más cómoda y fresca para el uso diario, un comedor para una mesa de doce puestos donde se sentara la familia con todos sus invitados; nueve dormitorios con ventanas hacia el patio y un largo corredor protegido del resplandor del mediodía por un jardín de rosas, con un pasamanos para disponer macetas de helechos y tiestos de begonias.

Dispuso ensanchar la cocina para construir dos hornos, destruir el viejo granero donde Pilar Ternera le leyó el porvenir a José Arcadio, y construir otro dos veces más grande para que nunca faltaran los alimentos en la casa. Dispuso construir el patio, a la sombra del castaño, un baño para las mujeres y otro para los hombres, y al fondo una caballeriza grande, un gallinero alambrado, un establo de ordeñar y una pajarera abierta a los cuatro vientos para que se instalaran a su gusto los pájaros sin rumbo.

Gabriel García Márquez. Cien años de soledad

(2007; 68).

Referirse al hábitat, específicamente al que adquiere un carácter residencial, por sustentar las relaciones íntimas de la familia, es referirse no solo al espacio de la vivienda, sino, al contexto físico y de relaciones que posibilita tejer de manera extendida la madeja de hilos que configuran las familias, y más tarde determinan el hogar.
Para poder analizar cómo las estructuras familiares configuran sus ambientes residenciales, es preciso enmarcar el concepto de familia dentro del aspecto cultural e histórico, para de esta manera, tener una referencia que permita comprender sus formas de habitar el espacio. Las diferentes formas que adoptan las viviendas son un complejo fenómeno, pueblos con distintos ideales, ambientes físicos, situaciones económicas y estructuras familiares.

El universo teórico que pretende sentar las bases de una realidad basada en la cotidianeidad y la experiencia, se vislumbra entre dos hechos claves: la influencia de los contextos geográficos y culturales en la definición de las instituciones familiares, sus formas de relación y sus modos de habitar $y$, por otro lado, el hecho cómo las transformaciones familiares, atendiendo a circunstancias externas e internas han modificado el contexto físico y funcional de su residencia, mediante sus representaciones de identidad del grupo familiar.

Este asunto, define un marco referente a tres elementos conceptuales fundamentales en este estudio, tomando como referente el vínculo entre el humano y el espacio:

\section{EL HABITANTE, EL HABITÁCULO Y SUS MODOS DE HABITAR.}

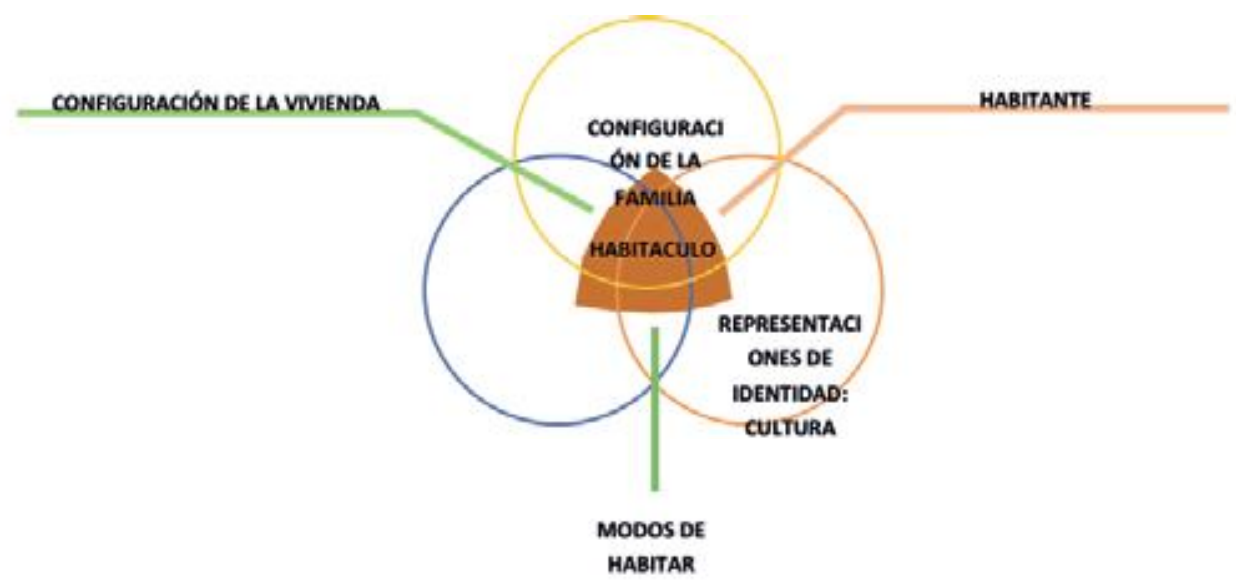

Figura 1. Elementos conceptuales del estudio. Fuente. Elaboración propia. 2014 


\section{EL HABITANTE Dimensiones ideológicas del cambio familiar}

El contexto en el que se enmarca el concepto de familia, no solamente está íntimamente relacionado con los cambios y transformación que estas han sufrido en el transcurrir del tiempo, sino, también por los fenómenos sociales, políticos, económicos y hasta biológicos que han permeado sus formas de instauración.

La familia es tratada desde el campo de la sociología como la red social primaria esencial en cualquier etapa de la vida y que sirve de intermediación entre el individuo y la sociedad.

Sin embargo, Gutiérrez De Pineda (2000) advierte que la caracterización de la familia se debe a las particularidades del contexto geográfico y cultural donde se construyen. Estos elementos inciden en pautas de comportamiento que se traducen en identidad, las cuales son muy importantes al momento de estudiar los cambios en las estructuras familiares.

En los estudios sobre la familia y el parentesco es necesario redibujar el concepto a la luz de los cambios demográficos, biológicos, culturales y sociales que acontecen en la actualidad, en donde la estructura familiar se ha apartado de la noción dominante de la década de los sesenta, según la cual la familia, específicamente la construida en los contextos colombianos, seguía patrones hispánicos y de la iglesia católica romana, como unidad inamovible e indisoluble. Esta generalidad deja de lado la conformación de estructuras familiares ampliadas bajo circunstancias como los casos de unión libre, la adopción y hasta la concepción cultural de la extensión familiar en el plano de las instituciones de poder como el compadrazgo, la amistad y el avecinado como producto de la llamada familia social, que más allá de los lazos de consanguinidad biológica.

Por otro lado se vislumbran fenómenos de tipo socioeconómicos y políticos que han influido de manera directa, sin embargo, no tan estudiada en las transformaciones familiares, como por ejemplo, los casos de desplazamientos forzados o voluntarios, la informalidad que trae consigo población flotante con capacidad reproductiva, el aumento del madre solterismo, la cohabitación y la alta tasa de natalidad actual, producto del auxilio económico conferido por políticas de Estado a las familias vulnerables, según la cantidad de niños por hogar que figuran en condiciones de pobreza.

Estas características, particulares del cambio, en sus distintos momentos, tiempos y ritmos resultan claves para entender la génesis de los conflictos entre los grupos humanos, con motivos de acomodación en el universo real de las cosas y sus contextos. Esto permite inferir que la caracterización de las familias colombianas encierra en sí un carácter polimorfo estructurado a partir de los cambios que se advierten a partir de las particularidades de su contexto geográfico y cultural, y de las tensiones que se generan en el seno de estas, entre las ideas antiguas y las nuevas, en el debate de la ideología y la tradición. (Gutiérrez De Pineda. 2000).

Essabido, entonces, queel modelo tradicional de familia conyugal está cambiando a un ritmo acelerado. De hecho, si lo definimos como el de la familia de los cincuenta, en la que el hombre se encargaba de trabajar afuera, la mujer organizaba el hogar y el cuidado de los niños y todos convivían en una vivienda separada de la parentela mayor, se constata que ese modelo dejó largamente de ser el mayoritario para convertirse en casi una excepción (Filgueira y Peri, 1993).

A pesar de la magnitud de los cambios reseñados, se sabe poco sobre las características de la población que incorpora esos cambios. Cuando se analiza, por ejemplo, la emergencia de nuevas formas de conyugalidad, como las uniones consensuales - la multiplicidad de parejas en el tiempo, se encuentra que estos comportamientos no tienen la misma prevalencia entre distintos sectores sociales. Las mujeres de sectores sociales bajos tienen mayor afinidad a convivir en pareja sin estar casadas, que las mujeres de sectores acomodados, al igual que respecto a formar otra pareja después de la disolución de la primera unión. 
PROCESOS URBANOS - Revista de divulgación científica vol. 2 enero - diciembre 2015 (107 - 116)

Contrariamente a la pauta antes descrita, las mujeres de los sectores sociales bajos son las más apegadas al sistema tradicional de división del trabajo, en el que la mujer se encarga exclusivamente del cuidado de los niños y de las tareas del hogar. (Peri. 2003).

Pero, fuera de ciertas tendencias estadísticas del tipo descrito en el párrafo anterior, es posible pensar que la adopción de nuevos comportamientos no es solo una adaptación diferencial a un entorno de privación o estrés, derivado de la articulación de la familia con otros órdenes sociales, sino el resultado de un cambio de valores y actitudes que lleva a esos nuevos comportamientos.

En realidad, esta perspectiva reduccionista de los valores no es exclusiva de la demografía. Lesthaeghe y Moore (1999) muestran cómo, en la explicación de la dinámica social, los valores han sido relegados también en otros varios cuerpos teóricos, como las teorías neoclásicas en economía, las teorías del intercambio en sociología, así como diversas versiones del marxismo. Sin embargo, el papel de la ideología y de los valores juegan un rol central en lo que Lesthaeghe llama factores ideacionales, es decir, las perspectivas de futuro tanto individuales del ser como las de la colectividad o grupo familiar estimulan el desempeño de nuevos roles dentro de la estructura de la familia y modifican los comportamientos y funciones tanto en tiempo como en espacio.

En esta misma línea de pensamiento es posible situar los pensamientos complejos que vinculan a la familia como un sistema de interacción, que amplía el campo de visibilidad hasta la toma de conciencia del papel de la familia dentro de la sociedad y el papel de la sociedad dentro de la familia, asunto que sitúa esta compleja institución como una unión de diversidades, en donde no solo caben en línea directa los progenies, sino una sociedad entera a partir del llamado interaccionismo social y cotidiano.

Los autores que confluyen en estas teorías de la interacción como HERBERT BLUMER, DAVID CHEAL, TURNER, KLEIN Y WHILE, entre otros, concluyen que la institución familiar, dentro de las transformaciones que ocurren tras los cambios generacionales, asumen una visión del mundo compartida a través de símbolos y significancias que posibilitan su unidad, sin embargo, esta visión puede estar en concordancia con la de otras personas a través de su interacción de cotidianidad, que no están dentro del grado de parentesco, pero sí de familiaridad, lo cual los hace figurar dentro de esta estructura, permitiendo la conformación de vínculos familiares extensos, asunto que debe tenerse en cuenta al momento de estudiar las dinámicas de la familias en el contexto social y físico, y de observar cómo esta nueva estructura familiar configura los espacios de sus hábitats residenciales, de manera que se suplan las necesidades de todos sus miembros.

Este asunto nos hace interpretar, que existen unas variables ideológicas importantes a tener en cuenta cuando se está frente a las transformaciones familiares actuales; una de ellas es el institucionalismo. La familia hoy, carece de este carácter sin dejar de ser familia con vinculación afectiva, es decir, no es necesario cumplir con requisitos legales impuestos para que el vínculo familiar esté activo en la organización familiar. Este asunto ha dado circunstancias repetitivas como el aumento del divorcio, la unión libre, el intercambio de roles parentales, la igualdad de género, etc. los cuales han influido de manera directa en la adopción de nuevos comportamientos familiares.

La familia es entonces un grupo humano complejo, permeado por los factores $y$ fenómenos que ocurren en el exterior de ellas, sin embargo, es y será siempre la manera más efectiva de vinculación, de la unidad y la diversidad, sin embargo, técnicamente en términos descriptivos y explicativos, la noción de familia ha sufrido un paso conceptual y teórico importante el cual no es posible omitir: el cambio de la idea de familia a la idea de hogar. 


\section{EL HABITÁCULO}

\section{La casa. El escenario de la vida familiar}

La gente, las personas que habitan los edificios, siguen siendo, en el fondo, los grandes olvidados de la arquitectura residencial.

Sin embargo, una casa es una vivienda más la gente que la habita y los objetos que guarda.

Xavier Monteys y Pere Fuertes. La casa collage

(2001: 14).

Hablar de casa no es hablar exclusivamente de vivienda. La casa se constituye en efecto de este estudio, como un espacio social de interacción, donde converge la unidad y la diversidad del habitar, un refugio de naturaleza espiritual y social, una transición entre lo público y lo privado.

La casa adquiere un concepto más amplio, de universalidad, atendiendo a las particularidades de las dimensiones humanas en donde se construye, las circunstancias sociales, económicas y culturales.

Así como la vivienda protege el desarrollo de las acciones y movimientos de la vida cotidiana: comida, descanso, sueño, trabajo, necesidades fisiológicas, etc. también adquiere un valor a juicio de sus habitantes, de los principales actos de su existencia, de los cuales ella es escenario obligado. Las edades de la vida que aquí se suceden y los ritos que perpetúan a la familia, la marcan con un sello de perennidad identificándola con el linaje. (Pezeu - Massabuau. 1989).

La casa tiene entonces, una de las cualidades más universalmente reconocidas: la capacidad de perpetuar la familia. Es el receptáculo duradero del fuego, del hogar.

La casa abre y sella el destino individual, es el lugar del nacimiento y por lo tanto el lugar de la muerte, reflejo psicofisiológico presente en el ser por una voluntad de permanencia del modelo espacial de carácter de refugio físico que mantiene su imagen al filo de las generaciones, más allá de la muerte individual. De este modo, todo parece apuntar a la imposibilidad de entender de forma separada la casa y la familia.

Una relación considerada generalmente armoniosa y necesaria entre la figura del hombre en reposo o en movimiento y la forma de su vivienda se da por igual en todas las civilizaciones. En este espacio hueco, el hombre desarrolla fácil y espontáneamente los movimientos de su vida cotidiana. Inscribe su cuerpo naturalmente en cada una de sus partes y ella responde con docilidad a las referencias de su sistema de orientación. (Pezeu - Massabuau. 1989).

Este asunto hace pensar que el ser humano, en su relación consigo mismo (individualidad) y con el otro (colectividad), configura el espacio que habita, hecho que incide directamente en el espacio de la vivienda, pues su diseño ${ }^{2}$ es una especie de diagrama que responde a los modos de vida de una familia. (Correa. 2007). Correspondiendo con lo planteado por Jacques Pezeu - Massabuau (1998), la vivienda, es el fruto de un modelado del comportamiento desempeñado a través de largos siglos de vida colectiva en el seno de una civilización específica.

Las nociones de comportamiento individual y colectivo forman un complejo indisoluble con la arquitectura de la casa.

En este plano, entra en juego la teoría del desarrollo familiar, en la medida en que se estudia en ciclo de vida de las familias, sus interacciones y las roles que cada uno de sus miembros desempeña. (Mattessich $Y$ Hill. 1987).

El ciclo de vida correspondería entonces, a los cambios que inciden en que se modifiquen las estructuras familiares, bien sea en cuanto a la edad, el número de miembros, sus oficios; lo que finalmente provocaría trasformaciones en el modo en que se han organizado los miembros de la familia para desarrollar sus actividades cotidianas y por ende su funcionamiento. (Gracia Y Musitu. 2000).

\footnotetext{
${ }^{2}$ Cuando se hace referencia al diseño, no necesariamente se hace alusión al ejecutado por un profesional de la arquitectura, sino más bien, a la autoproducción y organización propia de los espacios, con sus formas y sus funciones particulares.
} 
PROCESOS URBANOS - Revista de divulgación científica vol. 2 enero - diciembre 2015 (107 - 116)

Esta teoría resulta ser importante al momento de interpretar los cambios que han sufrido las familias y cómo esas cambios han configurado diferentes formas de habitar el espacio, transformándolo. Los estadios del desarrollo generan nuevos roles en la familia y agregan funciones y formas a los espacios donde habitan. El ciclo familiar origina consecuencias directas con respecto al comportamiento habitacional y engendra exigencias en relación con la vivienda.

La vivienda, la casa, el hogar, son denotación que el ser humano ha establecido a manera de cualificación del recinto arquitectónico que alberga y posibilita la interacción consiente e inconscientemente de la vida, tanto en la intimidad como en la colectividad.

Sin embargo, en la actualidad, pensar en la vivienda desde la perspectiva de hábitat, pone a consideración las intervenciones físicas y modificaciones arquitectónicas que este recinto ha padecido a nivel formal, funcional y estructural, dado el carácter objetual y mercantilista que el sistema económico y político vigente le ha impuesto a esta solución arquitectónica.

Enfrentar la vivienda al concepto de hábitat permite indagar por ella como parte de las redes que tejen distintos grupos humanos en conexión con su espacio, inherentes a la realización de la cotidianidad humana, en esferas tanto operativas y fisiológicas como sociales, económicas y simbólicas, superando con ello el sentido funcional que normalmente se ha atribuido a la vivienda, bien como valor de uso o de cambio, en tanto objeto, máquina o función.

Estas interpretaciones de los conceptos arquitectura y hábitat, se enmarcan en la perspectiva de los contextos sociales, culturales y geográficos que determinan a su vez las maneras de configurar el territorio y sus espacialidades en el marco de las dinámicas cotidianas de la sociedad, su idiosincrasia, sus representaciones de la realidad y su estructura social.

En el caso de la familia, su existencia no destruye las implicaciones territoriales de los individuos, sino que las integra (García en Gómez. 1994). Por tanto, el espacio arquitectónico es el sustrato que posibilita las interacciones sociales y que a su vez, es el conjunto de estos elementos de la estructura social los que cualifican el espacio como una expresión de sus hábitos, su cultura y sus tradiciones.

Visto así, la casa en clave de hábitat, es
una elaboración cultural y no un mero
espacio físico (Gómez. 1994), a través
de la cual se ponen en manifiesto
modelos propios de cada cultura, dando
lugar a la diversidad y a la significancia.

Para concluir, el ser humano requiere de la arquitectura, el espacio cualificado de la casa para alcanzar el morar, para aprender a habitar (Viviescas, 2012), sin embargo, esta configuración parte de nosotros mismos, del desarrollo de nuestro ser en interacción con el espacio en concordancia con nuestros modelos culturales y sociales. La casa en clave de hábitat, es un pequeño micro universo que hace posible la mirada hacia un mundo lo más gigantesco posible.

En conclusión, si la función pasiva de la casa es la provisión de un techo, su fin positivo es la creación de un entorno más adecuado al modo de vida de un pueblo; en otras palabras, es una unidad espacial social. (Rapoport. 1969).

La casa no es tan solo una estructura, sino una institución creada para un complejo grupo de fines. Porque la construcción de la casa es un fenómeno cultural, su forma y su organización están influidas por la circunstancia social a la que pertenece. (Rapoport. 1969).

\section{MODOS DE HABITAR}

La fuerza sociocultural en la configuración de la familia y la casa

Los estadios de familia y casa son ideales del ser humano en la realización de su ser enológicamente, en particular porque el hombre tiene una gran propensión a simbolizar todo lo que ocurre y a reaccionar ante los símbolos como si fuesen verdaderos estímulos ambientales, que se constituyen en la encarnación física de un ambiente ideal 
hecho visible. Estas idealizaciones reflejan visiones del mundo y modos de vida diferentes.

Esta discusión considera el impacto de la imagen cósmica y la naturaleza simbólica que cada cultura concibe en la configuración de sus lugares de habitar y su manera de relacionarse con ellos. El cosmos puede estar reflejado en un microcosmos a distintas escalas, el país, la ciudad, el pueblo, la casa, el espacio de la casa y los muebles y ornamentos que hay en él, que no son más que la impresión de la personalidad individual influida por el ideal colectivo, representaciones de identidad. Cada uno por separado o todos, pueden reflejar la forma en que se visualiza el mundo (Rapoport. 1969).

Este hecho saca a relucir la importancia de los valores tradicionales en la configuración de los espacios y en la manera como este espacio es aprehendido y apropiado por el ser humano. La carga simbólica de los movimientos en la cotidianidad, los utensilios, la tierra, están estrechamente relacionados al orden de la sociedad, el del pensamiento y el del universo.

\section{La casa es entonces según cada cultura, un modelo del universo a pequeña escala.}

Las bases teóricas que relacionan el fundamento de la estructura familiar con el contexto cultural y con el habitat donde residen han tenido un enfoque determinante e interdependiente, es decir, que la una es a la otra como la formación de un conjunto de interrelación. Dentro de las teorías más importantes para esta investigación se tienen:

Formas psicológicas de funcionalismo que relacionan las creencias y prácticas culturales con la personalidad de los individuos, y la personalidad del individuo con las creencias y las prácticas culturales. Entendiendo por personalidad, las formas pautadas de pensar, sentir y comportarse el individuo. (Gutiérrez. 2000).

Las transformaciones históricas que ha sufrido la estructura de la familia están dadas por tres factores fundamentales: el primero, el tipo de familia existente antes de la llegada de los españoles a las poblaciones indígenas, los cuales eran de carácter evolutivo y diverso, el segundo, la superposición a los indígenas de los principios morales cristianos mediante un proceso de aculturación hispánica, tercero, la enseñanza de la doctrina católica en los grupos criollos. (Gutiérrez. 2000).

En esta primera parte, la familia indígena era una familia extensa, en la que primaba el interés social sobre el individual al asumir la vida de pareja. La mujer ocupaba una posición destacada en el interior del hogar $y$, todos los miembros de la tribu eran considerados como parte de la familia, aunque no existieran rasgos de consanguinidad y se permitiera la reproducción entre ellos mismos. El sistema de parentesco indígena representaba un estatus, considerando que eran padres o madres de toda una generación, y se consideraban hijos todos los de la siguiente generación.

Este asunto, contrario al sistema descriptivo español, fue puesto en riesgo, lo que genera una nueva estructuración familiar y estableció nuevas instituciones católicas.

De aquí en adelante, es posible hallar otros factores ligados al proceso dinámico y global de moldeamiento de la familia en contextos sociales específicos, tales como, las consideraciones económicas actuales y modalidades de empleo, la informalidad, descenso en la natalidad, re-socialización de la familia, por ejemplo, adopciones, parejas homosexuales, monoparentales, (solteros, divorciados o viudos), hijos por interés, entre otros. Consecuencia de la construcción de nuevas identidades individuales y colectivas que surgen con la modernidad y las nuevas concepciones religiosas menos impuestas.

Sin embargo, esta nueva imagen de familia no indica necesariamente un debilitamiento de los lazos familiares, sino, por el contrario, un fortalecimiento de otras formas de vinculación más allá de la filiación. Índices estadísticos sugieren el paso de un modelo conyugal hegemónico basado en el matrimonio a la emergencia de nuevas formas de relación y convivencia mucho más diversificadas. 
PROCESOS URBANOS - Revista de divulgación científica vol. 2 enero - diciembre 2015 (107 - 116)

Dentro de estas nuevas formas de integración familiar, es posible estudiar aquellas cuya constitución inicia en los clanes y grupos étnicos indígenas $y$ negroides y que ha sido heredado por el complejo cultural en donde se asentaron mayormente estas etnias, como las riveras y las costas. El modelo familiar polisémico abarca entonces gente cuya afinidad individual teje redes informales de relación con una constante afectividad y preferencia del desarrollo personal y libertad voluntaria. Por ejemplo, los amigos más cercanos, los vecinos, y personas con parentescos de afinidad.

\section{EN CONTEXTO CARIBE: Sincelejo. Familia ampliada, vivienda extendida}

En contexto caribe, la familia sufrió la resistencia de su mezcla étnica ante la imposición hispánica en la compleja aceptación de la institucionalidad de la iglesia católica.

Este hecho constata a las familias caribes como organizaciones de afecto y vinculación cosmogónica, afín a la manera de pensar y de sentir. Asunto que amplía el panorama de la consanguinidad y el parentesco.

El territorio caribe es la casa, y la casa es la totalidad del territorio, lo que no se hace en el interior trascurre en la dinámica de lo público, la calle se conjuga y se convierte en un espacio festivo, de intercambio, en el perfil de una vivencia caribeña. El Caribe es una gran familia, repleta de recuerdos, pero dispuesta a un porvenir a partir de la construcción de su hábitat en su vivencia cultural.

Emerger en una costa en donde las fronteras visuales se configuran en el horizonte del mar, el mismo, que en el ciclo del día entra en contacto con el sol y con la luna, como queriendo adueñarse del firmamento, es estar frente a una cultura que abre sus brazos y expresa en su identidad un universo sin límites.

Los habitantes de la costa son protagonistas del mundo macondiano de García Márquez y conforman un conjunto social permeado por el dolor, el atropello y el odio. Sin embargo, con la fuerte brisa que proviene del océano se van estos vestigios de sufrimiento $y$ emerge una sociedad marcada por la alegría y la fiesta, concebida esta última no como sinónimo de bullicio sin sentido, sino como expresión de la personalidad extrovertida del caribeño y como el pretexto para enmascarar el olvido, en el marco de una conjugación de razas aborígenes, africanas y españolas, sumando las inmigraciones árabes del último tercio del siglo XIX.

Todos estos aportes han dado origen a una idiosincrasia propia, única. Capaz de reconfigurar sus modos de vivir y de habitar, sus relaciones consigo mismo y con el otro.

El habitante caribe es habitante del mundo, su apertura les hace albergar al otro en su casa grande, su aldea, su universo.

Los estudios de familia y sus cambios en las configuraciones a lo largo del tiempo y de las circunstancias de globalización y el régimen económico social imperante, han sido enfocados después de múltiples cambios, desde la antropología social y la perspectiva etnográfica, en consecuencia se puede analizar que los nuevos modelos de familia, no dependen tanto de su inamovible composición de estructura genealógica, como del contexto social y cultural en que se conforman.

La familia y sus modos de comportamiento están íntimamente ligados y condicionados por el desarrollo y equilibrio de la sociedad, constituyéndose así en la red social primaria para el ser humano en cualquier etapa de la vida.

Gutiérrez de Pineda (2000) en su obra Familia y cultura en Colombia, advierte que la caracterización de la familia colombiana adquiere su carácter a partir de los cambios y particularidades del contexto geográfico y cultural de donde provienen y de las tensiones de sus ideas de tradición y sus nuevos ideales, lo que quiere decir, que una estructura familiar marcada culturalmente adquiere unas forman de comportamiento, expresión y representación particulares que generan la diversidad con las estructuras de otras latitudes. 
La importancia entonces, del estudio de la familia radica en la comprensión del cambio a nivel social e institucional que esta ha tenido a lo largo del tiempo y cómo se han configurado sus distintos modos de habitar el territorio y de instaurar nuevas redes sociales familiares.

Este hecho encuentra correlación con lo directamente observable: la casa, concebida según Levi-Strauss como una unidad social que teje redes sociales. Este interés por el hábitat familiar desentraña aún más las características homogéneas y lineales de la "Sagrada familia" en su calidad biológica y pone a consideración aspectos de convivencia y sociabilidad visibilizados en los aspectos rutinarios y de la vida cotidiana.

La casa caribe permanece abierta, queriendo espantar el calor intenso que su condición geográfica le otorga. Este hecho posibilita su flujo constante de entradas y salidas, del que llega al encuentro de su territorio, del que se va al descubrimiento de otros, pero sin perderse, del que llega a la extensión de su familia, del que arriba pidiendo ayuda y que se queda porque lo encontró todo. Aquí se tejen los micro universos, en medio de las diferencias y los sentidos de posesión del espacio, el espacio íntimo de la casa, del hogar, de la familia, del ser.

... La forma como se recibe al vecino y su grado de familiaridad permite que acceda al salón o que penetre hasta la cocina o la alcoba; la actitud que se asume cuando los nietos llegan a casa de la abuela... (Gómez. 1994).

Esta descripción adquiere un carácter profundo en la interpretación del concepto de familia, y especial connotación para el habitante del Caribe colombiano, y de igual forma para el valor que en él adquiere el símbolo de la casa, su forma y su función, visto como un universo simbólico y no como un hecho denotativo de mera solución a una necesidad. El carácter connotativo que adquiere el hábitat residencial está basado en las prácticas cotidianas de sus habitantes, sus acciones de vivir y de habitarlo, impregnándolo de signos particulares personalizadores que permiten al habitante tomar posesión de su mundo y compartirlo con el otro, en su realidad del presente y sus aspiraciones de futuro.

La familia configura su espacio para habitar, sin embargo, el mercado inmobiliario en su afán mercantil y utilitarista vende modelos residenciales sin considerar las fundamentaciones de las estructuras sociales y culturales que se encuentran inmersas en los núcleos familiares del siglo $X X$.

Consecuencia de esto, viviendas reducidas y familias hacinadas. La casa debe salirse de sus paredes y debe encontrar sus espacios en los contextos públicos del vecindario, del barrio y la ciudad; sacan la tradición a la calle, no habitan la casa, la toman como un elemento para pernoctar; la cultura no se resiste al límite, sin embargo la economía y el régimen la someten.

\section{REFERENCIAS}

Arango, G. (1997). La poética de la casa de patio a la casa moderna. Medellín. Edit. Universidad Nacional.

Arango, G.; Pelaez, P.; Wolf, G. (1997). Los cambios en la vivienda. Discursos y percepciones. Medellín. Edit. Universidad Nacional.

Barrios, M. (2010). Apuntes sobre familia y cambio social. Revista Barrios. Barranquilla. Observatorio de medios y opinión pública, Universidad del Norte. Pag. 163-179.

Berger, P.; Luckmann, T. (1994). La construcción social de la realidad. Buenos aires. Edit. Amorrortu.

Bronferbrenner. U. (1989). The ecological systems theory. Grenwich. Edit. Press.

Bubolz, M.; Sontang, S. (1993). Teoría de la ecología humana. Nueva York. Edit. Press.

Correa, J. (2007). Incidencia cultural, económica y del tamaño de la familia en la vivienda de interés social. Bogotá. Edit. Universidad Nacional de Colombia. 
PROCESOS URBANOS - Revista de divulgación científica vol. 2 enero - diciembre 2015 (107 - 116)

Fals Borda, O. (1979). Historia doble de la costa. 1ra edición. Bogotá. Edit. El Ancora.

Galindo, C., J. (1998). Técnicas de investigación en sociedad, cultura y comunicación. Mexico. Edit. Addidon. Pag. 523.

Gelles, R. (1995). Familias contemporáneas. Una revisión sociológica. Londres. Edit. Sage.

Gracia Fuster, E.; Musitu Ochoa, G. (2000). Psicología social de la familia. España. Editorial Paidos.

Gubrium, J.; Holstein, J. (1990). ¿Qué es la familia? View Monuntain.

Gubrium, J.; Holstein, J. (1993). Fenomenología, etnometodologia y discursos familiares. Nueva York. Edit. Press.

Gutierrez De Pineda, V. (2000). Cultura y familia en Colombia. Tipologías, funciones y dinámicas. Medellín. Edit. Universidad de Antioquia.

Klein, D.; While, J. (1996). Introducción a las teorías de familia. Londres. Editorial sage.

Mattessich, P.; Hill, R. (1987). Ciclo vital y desarrollo de la familia. Boston. Edit. Houghton.

Mayol, P. Certeau, M. Giard, Luce. (1999). La invención de lo cotidiano II: habitar, cocinar. México. Edit. Universidad Iberoamericana.

Meisel, A.; Perez, G. (2006). Geografía física y poblamiento en la costa Caribe colombiana. Cartagena. Edit. Banco de la Republica.
Munné, F. (1996). Entre el individuo y la sociedad. Teorías actuales sobre el comportamiento interpersonal. Barcelona. UEB.

Muñoz, A. La vida cotidiana o ¿dónde fue que nos perdimos? En, http:// www.filosofia.mx/index.php?/perse/ archivos/la_vida_cotidiana_o_donde_ fue_que_nos_perdimos. España. 2011.

Paternina, A. (2001): Sincelejo: ayer y hoy. Sincelejo. Edit. Banco de la República.

Rapoport, A. (1972). Vivienda y cultura. Milwaukee. Edit. Universidad de Wisconsin.

Saldarriaga, A. (1982). Calidad y habitabilidad de la vivienda. Bogotá. Edit. INJAVIU.

Schmidt-relenberg, N. (1976). Sociología y urbanismo. Madrid. Edit. Nuevo urbanismo.

Segalen, M. (1992). Antropología histórica de la familia. Madrid. Editorial Taurus.

Sierra, J. (2013). Aldea y universo un perfil de la cultura popular. En: el tono azul del Caribe colombiano. Sincelejo. Edit. Multigráficas.

Sierra, J. (2013). Perfil de una vivencia caribeña. En: el tono azul del Caribe Colombiano. Sincelejo. Edit. Multigráficas.

Smith, S. (1995). Familia y estudios multiculturales. Nueva York. Editorial Press.

Turner, R. (1962). Construyendo roles. Procesos vs conformidad. Nueva York. Editorial wiley. 